\title{
An Elementary 1-Dimensional Model for a Solid State Lithium-Ion Battery with a Single Ion Conductor Electrolyte and a Lithium Metal Negative Electrode
}

\author{
M. Mykhaylov ${ }^{1}$, M. Ganser ${ }^{2,3}$, M. Klinsmann², F.E. Hildebrand ${ }^{2}$, I. Guz $^{1}$, \\ and R.M. McMeeking ${ }^{1,4,5,6}$ \\ ${ }^{1}$ School of Engineering, Aberdeen University, King's College, Aberdeen AB24 3UE, Scotland \\ ${ }^{2}$ Robert Bosch GmbH, Corporate Sector Research and Advanced Engineering, 70049 Stuttgart, \\ Germany \\ ${ }^{4}$ Materials Department, University of California, Santa Barbara CA 93106, USA \\ ${ }^{5}$ Department of Mechanical Engineering, University of California, Santa Barbara CA 93106 \\ ${ }^{6}$ INM - Leibniz Institute for New Materials, Campus D2 2, Saarbrücken, Germany
}

Dedicated to Professor Norman A. Fleck on the occasion of his $60^{\text {th }}$ birthday.

\begin{abstract}
An elementary 1-dimensional model is developed for a solid state lithium-ion battery having a single ion conductor electrolyte, a lithium metal negative electrode and a composite positive electrode. The battery topology is assumed to be of the layered variety, thereby justifying the 1dimensional formulation. The governing equations for the electrochemical kinetics at the interface between the negative electrode and the electrolyte separator are stated, as are those for ion transport in the electrolyte. The positive electrode is assumed to be a particulate composite of storage material within a matrix of electrolyte. A mixture theory is developed for the positive electrode encompassing ion transport in the electrolyte matrix and storage and unstorage of lithium in the active material subject to electrochemical kinetics at the perimeter of the storage particles. Many simplifying assumptions are made with the advantage of them leading to closedform or semi-closed-form solutions, including linearization of the equations governing the redox kinetics at interfaces in the battery between electrolyte and active material. An approximation is given for the concentration of lithium in the positive electrode of the battery during discharge, with the details depending on a length scale parameter that depends on the competition between the rate of lithium insertion into/extraction from the storage particles and the rate at which lithium ions are transported in the electrolyte. When the conductivity of the electrolyte is high and the redox reactions are relatively sluggish, this length scale parameter is comparable to the thickness of the positive electrode or larger than it. In that case lithium insertion into/extraction from storage particles occurs everywhere within an active zone of the positive electrode, but with the rates least at the current collector of the positive electrode. If the conductivity of the electrolyte is poor and the redox reactions rapid, the length scale for the solution is small compared to the thickness of the positive electrode and insertion into/extraction from storage particles occurs only in a narrow slice of the positive electrode. This slice moved along the positive electrode and separates a region of it that is completely filled/empty from a region of it that has not yet gained or lost any of its lithium. In all cases there will also be a region of the positive electrode near the separator that is completely filled during discharge and completely
\end{abstract}


empty during battery charging. Our results also give outcomes from which the internal resistance of the battery can be estimated.

\section{Introduction}

Solid state batteries have potentially useful characteristics, such as a high specific capacity, superior electrochemical stability in some contexts, and the ability to be exploited as structural elements in transportation systems, thereby saving vehicle weight compared to liquid electrolyte batteries [1]. Models for the performance of solid state lithium-ion batteries are under active development, with the objective of bringing insight into how such systems can be improved and optimized for capacity and durability [2-5]. Other treatments of the topic were placed in the literature some time ago [6,7], and are being updated as interest in solid state batteries rises [8]. Descriptions of the performance of lithium-ion batteries, their electrochemistry and the lithiumion and electron transport that takes place in them can be found in various papers and treatises including [9].

A complex feature of any lithium-ion battery is the positive electrode or cathode. It must consist of electrolyte to convey the lithium ions, storage material to hold the lithium, and a conducting path for electrons. In addition, the positive electrode must be held together, whether by a polymer binder or by direct bonding. As a consequence, the geometry of the positive electrode is usually complex and its topology complicates the development of models for its electrochemical behavior and for the transport of ions and electrons within it. Solid electrolytes themselves can also bring complications if they are binary ion conductors, in which case the counterions are mobile as well as the lithium ions. A consequence of this situation is that transport in the electrolyte must be modeled taking multi-component motion and interaction into account, just as in a liquid electrolyte [9]. Such considerations apply also to the separator between the negative electrode and the positive one, a homogeneous slab of electrolyte.

To avoid some of the challenges that are associated with modeling the positive electrode and the electrolyte we make simplifying assumptions for our elementary analysis. For the positive electrode we develop a mixture theory, and we restrict our attention to electrolytes that are single ion conductors in which only the lithium ions are mobile. In addition, we treat the battery in a 1dimensional manner, so that the problems to be solved generate relatively simple differential equations. We make other simplifying assumptions that are of a less sweeping nature, and that will be identified below.

One-dimensional models for battery function and performance are fairly common; perhaps the most prominent one is Dualfoil, based on the work of Newman and collaborators [10-12]. This computer algorithm solves the nonlinear coupled equations characterizing the battery cell, and is based on porous electrode theory, a mixture theory similar to that which will be used in the present treatment. Purkayastha and McMeeking [13] developed a 1-dimensional linearized model for lithium-ion batteries with a liquid electrolyte coupling 2 porous electrodes. The present treatment bears some similarity to the work in [13], but is focused on solid state batteries with a lithium metal negative electrode and a solid electrolyte. 
Given the 1-dimensional nature of our model, we depict the battery in Figure 1 where various components of the system are identified and dimensions specified. As can be seen in Figure 1, the battery is taken to have the negative electrode on the left and the positive electrode on the right, with a separator in between. The negative electrode is lithium metal, whereas the positive electrode, as noted above, is a composite of storage material, electrolyte, conducting material for electrons, and, possibly, a binder. A current collector is attached to the right-hand end of the positive electrode. The thickness of the positive electrode is $H$, which is taken to be constant on the assumption that any swelling of the storage material due to lithiation is small, i.e. it involves only an infinitesimal volume strain. Woodford, Carter and Chiang [14] have compiled values for lithiation volume strains for many storage materials used in positive electrodes. That information shows that many of those materials experience small volume changes upon lithiation, justifying our neglect of swelling in the positive electrode. In addition, depth of charge and discharge is usually limited to much less than the full capacity of the storage material, reducing the volume strains that the positive electrode experiences.

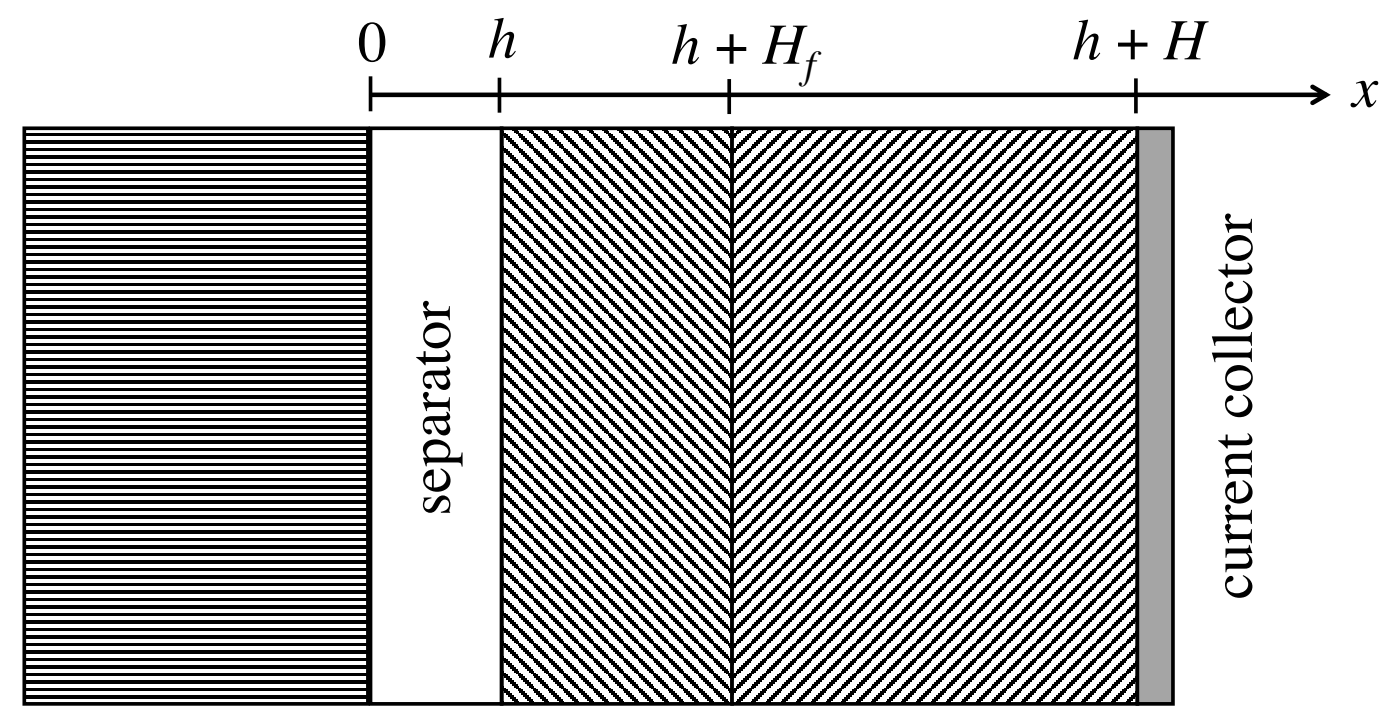

-ve electrode

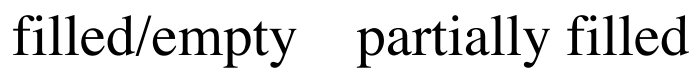
+ve electrode

Figure 1: A segment of a 1-dimensional battery showing the negative (-ve) electrode, the separator, and the positive (+ve) electrode. A coordinate, $x$, is also shown with the thicknesses of the 3 components indicated. The positive electrode consists of 2 segments. One segment is completely filled with or completely devoid of stored lithium molecules. The other segment is only partially filled. Note that the origin of the coordinate system is at the interface between the negative electrode and the separator.

The thickness of the negative electrode is unspecified as it varies depending on the state of charge of the battery. That is, when the battery is fully charged there will be little lithium in the positive electrode and the negative electrode, containing most of the lithium, will be thick. In a 
fully discharged battery most of the lithium will be in the positive electrode and the negative electrode will be thin. We note that the designation thick and thin in this description is relative, as the change of thickness may be small if the storage capacity of the positive electrode is small, limiting how much lithium can be added to and subtracted from the negative electrode.

Nevertheless, we point out that if $H$ in Figure 1 is large, the positive electrode will be able to harbor much lithium even if its swelling strain is small, and therefore the negative electrode will be then required to thicken much during charging and thin much during discharge.

The separator is purely electrolyte and has a constant thickness $h$. The thickness of the current collector is unspecified and is immaterial to the analysis. Note that the origin for the coordinate $x$ is at the interface between the negative electrode and the separator, thereby obviating any change of coordinate position of the interfaces between the separator and the positive electrode and between the positive electrode and the current collector. For operation of the battery there will be electrical leads attached to the negative electrode and to the current collector. These are not shown in Figure 1, but throughout we consider the wires to be connected to the left-hand end of the negative electrode and to the current collector.

Note that the positive electrode is divided into 2 segments. One is designated to be fully filled with lithium or empty, whereas the other segment is only partially filled. When the battery is discharged, lithium travels from the negative electrode through the separator to the positive electrode. Lithium reaches storage material in the positive electrode near the separator sooner and, in greater amounts, than for the storage material that is far from the separator. As a consequence, and as will be seen below, storage material in the positive electrode near the separator fills to saturation with lithium sooner than storage material that is further away from the separator. Thus, for discharging of the battery, we identify a segment of the positive electrode of thickness $H_{f}$ that is already filled with lithium in this way. We note that the parameter $H_{f}$ is a variable that progressively enlarges as the battery discharges. In addition, we point out that lithium ions will still be transported through the electrolyte in this segment. For similar reasons, during charging of the battery the opposite will happen; namely a segment of the positive electrode near the separator will be depleted of stored lithium. This feature is shown, for battery charging, by the empty designation in Figure 1. This segment will grow in thickness during charging, so $H_{f}$ is also a variable in this circumstance. Note however that lithium ions will still be transported through the electrolyte in this region, so it is not completely devoid of lithium ions even though it is devoid of lithium molecules in the storage material.

\section{Governing equations}

We now address the equations and associated parameters governing the electrochemical and transport phenomena that take place within the battery.

Current density We designate the current density within the battery to be $i$, defining the uniform flux of positive charge in the positive direction of $x$ within each of the components, i.e. in the negative electrode, in the separator, and in the positive electrode. This current density also applies at the interfaces, i.e. at those between the negative electrode and the separator, between the separator and the positive electrode, and between the positive electrode and the current collector. The uniformity of the flux is required by conservation of charge. When the battery is 
being discharged, $i>0$ and when it is being charged, $i<0$. The battery plus the external wire and devices connecting the negative electrode to the current collector form a current loop, so that the current in the external wire and devices is $I=i A$, where $A$ is the cross-sectional area of the battery. This current in the external wire and devices, carried solely by electrons, is directed from the current collector to the negative electrode when $i>0$, i.e. during battery discharge. The current in the negative electrode is also carried solely by electrons. In contrast, the current in the separator is carried solely by lithium ions, as is the case for the portion of the positive electrode that is either completely filled or completely depleted of stored lithium. In the remainder of the positive electrode the current is carried by a combination of electrons and lithium ions. The fraction supported by each is a function of position, $x$, as will be seen below.

We assume that the current density is held constant during charging and during discharge.

Negative electrode We define the electric potential as $\Phi(x, t)$ and choose $\Phi(0, t)=0$ in the negative electrode, serving as a datum. As noted above, the negative electrode supports a current density $i$ consisting of an electron flux. Ohm's law therefore requires there to be an electric field in the negative electrode in proportion to the current density and proportional to the resistivity of lithium metal. However, this electrode, being metallic, is a good electron conductor, and, in comparison to other potential drops and losses in the battery, the difference in electric potential across the negative electrode will be small. We therefore approximate the potential in it to be uniform and thus zero everywhere.

Interface between the negative electrode and the separator At this location a redox reaction takes place separating lithium molecules into lithium ions and electrons or reuniting them to form metallic lithium. During discharge of the battery, lithium metal is converted by the redox reaction into a lithium ion plus an electron, with the lithium ion passing into the separator and the electron transported to the left in Figure 1 and thus through the negative electrode. During charging of the battery, lithium ions arriving at the interface via the separator are combined with electrons arriving through the negative electrode. The resulting metallic molecules of lithium are plated onto the negative electrode at the interface. We note that we assume that this interface is impermeable to electrons.

We simplify the model by assuming that the interface between the negative electrode and the separator remains planar at all times. This assumption rules out the formation of dendrites of lithium in the separator $[6,7,15]$ and eliminates the possibility of the presence or the growth of non-planar imperfections on the surface of the lithium metal [3-7, 15]. In addition, we neglect any contribution made by mechanics to the rate at which the redox reaction proceeds [3-8]. As a consequence of these two assumptions, we can state the rate of the redox reaction, given by the Butler-Volmer equation $[9,16]$, as

$i=i_{o}^{a}\left[\exp \left(\frac{\alpha_{a a} F \eta_{a}}{R T}\right)-\exp \left(-\frac{\alpha_{a c} F \eta_{a}}{R T}\right)\right]$

where $i$, is the current density passing through the interface, $i_{o}^{a}$ is the exchange current density for the negative electrode/electrolyte interface, $\alpha_{a a}$ and $\alpha_{a c}$ are, respectively, the dimensionless anodic and cathodic symmetry coefficients for that interface, both positive, $F$ is Faraday's constant, $R$ is the gas constant, and $T$ is the absolute temperature. The parameter $\eta_{a}$ is defined as 
$\eta_{a}=\Phi^{\text {anode }}(0, t)-\Phi_{a}^{\text {elyte }}-U_{a}=-\Phi_{a}^{\text {elyte }}-U_{a}$

and is the surface overpotential for the interface where $\Phi^{\text {anode }}(x, t)$ is the electric potential in the negative electrode at time $t$, and thus assumed to be zero, $\Phi_{a}^{\text {elyte }}$ is the electrical potential in the electrolyte adjacent to the interface and $U_{a}$ is the potential difference across the interface at equilibrium, also known as its open circuit potential. The interface open circuit potential is related to lithium chemical potentials by

$F U_{a}=\mu^{\text {elyte }}-\mu^{\text {anode }}$

where $\mu^{\text {elyte }}$ is the internal chemical potential of lithium ions in the electrolyte and $\mu^{\text {anode }}$ is the chemical potential of lithium in the negative electrode [16]. The internal chemical potential excludes the electrostatic contribution given by the charge on the ion times the electric potential. It is common in the electrochemical literature to define the internal chemical energy to be the chemical potential, and to rename the total chemical potential as the electrochemical potential [9, 16]. We will abide by this convention. We note that both $i_{o}^{a}$ and $U_{a}$ are invariant, because the conditions of both the metal in the negative electrode and the electrolyte do not vary during the redox processes.

A comment on notation is appropriate at this juncture. We will introduce a Butler-Volmer equation for the storage material/electrolyte interface in the positive electrode, with the necessity of distinguishing its parameters from those in Eq. (1) \& (2). To enable this, we have liberally used the subscript or superscript $a$ for anode in Eq. (1) \& (2), doing so to avoid the clumsy use of the negative sign as a subscript or superscript. We use $a$ as the subscript or superscript because the negative electrode is often termed the anode in the lithium-ion battery literature. This terminology arises because, during discharge of the battery, the negative electrode is the anode. The negative electrode is cathodic during charging of the battery, and for that reason we avoid the terminology anode and cathode for the electrodes, other than using $a$ as the subscript or superscript designating the negative electrode, and $c$ as the subscript or superscript designating the positive electrode.

At this point, we will digress to characterize the significance of the various parameters in Eq. (1) $\&$ (2). We will describe their role for the interface between the negative electrode and the separator, but the reader should bear in mind that the equivalent set of parameters exists for the interface between the storage material and the electrolyte in the positive electrode.

The current density, $i$, passing through the interface is carried by lithium ions, and thus

$i=F j_{a}$

where $j_{a}$ is the molar flux of lithium ions out of the negative electrode, i.e. the number of moles of lithium ions passing out through unit area of the interface in unit time. When the current density is negative, lithium ions are passing from the electrolyte to the negative electrode. 
The exchange current density, $i_{o}^{a}$, is a one-way current that prevails at equilibrium when the surface overpotential, $\eta_{a}$, is zero. However, the net current density, $i$, is then zero because there is a current density $i_{o}^{a}$ driven from the negative electrode to the separator and a current density of the same magnitude driven from the separator to the negative electrode [16]. In other words, $i_{o}^{a}$ represents the rates of forward and backward redox reactions at the interface at equilibrium, with the forward and backward rates of reaction canceling each other out. Note that in equilibrium, with the net current zero, the potential difference, $\Phi^{\text {anode }}(0, t)-\Phi_{a}^{\text {elyte }}$, between the negative electrode and the separator is equal to $U_{a}$, the open circuit potential of the interface. The open circuit potential of the interface is thus its equilibrium potential difference, associated with zero net current across the interface. We refer to Eq. (3) and note that, once divided by $F$, the open circuit potential is equal in magnitude to the difference in chemical potentials of the lithium in the negative electrode and in the electrolyte. This gives us a clue as to what is happening at equilibrium. The chemical potential is driving the redox reaction in one direction, and the electric potential difference is driving it in the other, with the two effects canceling each other out [16].

If the surface overpotential is positive, i.e. the potential difference, $\Phi^{\text {anode }}(0, t)-\Phi_{a}^{\text {elyte }}$, between the negative electrode and the separator exceeds $U_{a}$, the magnitude of the first term in the bracket in Eq. (1) exceeds that of the second term and there is a net current of lithium ions from the negative electrode to the separator. This is the condition in which the negative electrode is anodic. If the opposite is true, and the surface overpotential is negative, i.e. the potential difference, $\Phi^{\text {anode }}(0, t)-\Phi_{a}^{\text {elyte }}$, between the negative electrode and the separator is less than $U_{a}$, the magnitude of the second term in the bracket in Eq. (1) exceeds that of the first term and there is a net current of lithium ions from the separator to the negative electrode. This is the condition in which the negative electrode is cathodic.

As noted above, the parameters $\alpha_{a a}$ and $\alpha_{a c}$ are dimensionless anodic and cathodic symmetry coefficients for the interface between the negative electrode and the separator. They determine the sensitivity to the interface electric potential difference of the anodic and cathodic contributions to the redox reactions at the interface. Theoretically they should sum to unity [9, 16], but, on an empirical basis, they are often taken to be independent.

Separator As noted above, we restrict ourselves to single ion conducting electrolytes, in which only the lithium ions are mobile. Thus, the counterions are firmly held by the material lattice and do not move. We also assume that the electrolyte is a poor conductor of electrons to the extent of being a perfect resistor for them. We also invoke charge neutrality in the electrolyte, known to be a good approximation to reality in almost all circumstances $[5,9,16]$. As a consequence, the concentration of lithium ions in the electrolyte is uniform, matching that of the immobile counterions. As a result there is no concentration gradient driven component to the motion of the lithium ions, and Ohm's law governs their transport in the separator so that

$i=-\chi \frac{d \Phi}{d x}$

where $\chi$ is the conductivity of pure electrolyte. 
Continuity of the electric potential requires that, in the separator,

$\Phi(0, t)=\Phi_{a}^{\text {elyte }}$

As a result, we can immediately integrate Eq. (5) to obtain, for the separator,

$\Phi(x, t)=\Phi_{a}^{e l y t e}-\frac{i x}{\chi}$

Thus, at the interface between the separator and the positive electrode

$$
\Phi(h, t)=\Phi_{a}^{e l y t e}-\frac{i h}{\chi}
$$

Interface between the separator and the positive electrode We consider the positive electrode to be a composite material of storage particles within a matrix. The matrix consists of electrolyte, material, usually carbon black, forming a conducting path for electrons and, possibly, a binder. As a consequence of charge conservation, the current density is continuous at this interface, a condition that we have already explicitly imposed by specifying a uniform current density throughout the battery. In addition, continuity of the electric potential requires it to be given, in the electrolyte at this interface, by Eq. (8).

Positive electrode As described above, the positive electrode is assumed to be a composite material of storage particles within a matrix, where the matrix consist of electrolyte, material that conducts electrons and, possibly, a binder. The positive electrode has 4 important attributes. One is its conductivity for lithium ions, determined by the characteristics of the electrolyte in the matrix. The $2^{\text {nd }}$ attribute of importance is the conductivity for electrons, usually provided by a coating of carbon black around the storage particles. The $3^{\text {rd }}$ is the capacity of the storage material for containing lithium, and the $4^{\text {th }}$ attribute is the electrochemical behavior of the internal interfaces in the positive electrode between electrolyte and storage material. We consider each of these in turn.

We first stipulate that we assume that the diffusion distances within the storage material in the positive electrode are short, and that the lithium quickly equalizes its distribution within any given storage particle. That is, we assume that the process of diffusion of molecular lithium within the storage particles is not rate limiting. Thus, we address the redox reaction kinetics at the surface of the storage particles, but neglect the transport of lithium within the particles, other than assuming that it is fast.

The potential $\Phi(x)$ within the positive electrode is the value it has in the electrolyte. The current density sustained by lithium ions is designated $F j$, where $j=j(x, t)$ is their molar flux. Due to the characteristics of the electrolyte, as described in the paragraph discussing the separator, Ohm's law gives

$F j(x, t)=-\chi_{c} \frac{\partial \Phi}{\partial x}$ 
where $\chi_{c}$ is the ionic conductivity of the composite positive electrode. We can expect that $\chi_{c}<$ $\chi$ due to a reduced cross-sectional area of electrolyte and, e.g., tortuosity. However, if there is not much binder present, and if the storage particles provide pathways for lithium molecular flux that contribute to lithium ion transport, then $\chi_{c}$ could be close in value to $\chi$. However, a limitation to this effect is the fact that ions taking this path would pass through 2 redox reactions, one upon entering a storage particle and one upon leaving it. Such reactions involve losses and therefore the effective conductivity associated with the path just described may not be very good. Although composite models for ion conductivity in particulate composites exist [17-19], we will not attempt to invoke any, and leave $\chi_{c}$ as a parameter. It could be measured experimentally in a positive electrode that is saturated with lithium in its storage material.

In the segment of the positive electrode that is either full of lithium or empty of it, i.e. within $h \leq$ $x \leq h+H_{f}$, the ionic current density is uniform and there is no electron flux. This situation arises because there is no redox reaction taking place within that segment, and the separator is a perfect insulator for electrons. Thus, $F j=i$ within that segment, and integration of Eq. (9) provides

$\Phi(x, t)=\Phi_{a}^{e l y t e}-\frac{i h}{\chi}-\frac{i(x-h)}{\chi_{c}}$

As a consequence, the potential at the right-hand end of the full/empty segment of the positive electrode is

$\Phi\left(h+H_{f}, t\right)=\Phi_{a}^{e l y t e}-\frac{i h}{\chi}-\frac{i H_{f}}{\chi_{c}}$

Both the electric potential in the electrolyte and the current density are continuous at this interface.

The potential, $\Phi_{s}(x, t)$, in the storage material in the positive electrode is determined by the electron conducting material that is presumed to coat the surface of storage particles. We note that charge conservation requires that the current supported by electrons in the positive electrode is $i-F j(x, t)$. We assume that the conducting path for electrons has conductivity $\chi_{e}$, and Ohm's law then gives us

$i-F j(x, t)=-\chi_{e} \frac{\partial \Phi_{s}}{\partial x}$

The effective electronic conductivity of the positive electrode is measurable experimentally. The boundary condition for Eq. (12) is that both sides of the equation must be zero at $x=h+H_{f}$, i.e. at the right-hand end of the full/empty segment of the positive electrode. This condition prevails because electrons cannot cross this boundary since there is no redox reaction to the left for them to participate in. Thus

$\frac{\partial \Phi_{s}\left(h+H_{f}, t\right)}{\partial x}=0$ 
and

$j\left(h+H_{f}, t\right)=\frac{i}{F}$

We now combine Eq. (9) \& (12) to obtain

$\chi_{c} \frac{\partial \Phi}{\partial x}+\chi_{e} \frac{\partial \Phi_{s}}{\partial x}=-i$

which gives us, with Eq. (11) taken into account,

$\Phi_{s}(x, t)=\Phi_{s}\left(h+H_{f}, t\right)+\frac{\chi_{c}}{\chi_{e}}\left(\Phi_{a}^{\text {elyte }}-\Phi\right)-\frac{i(x-h)}{\chi_{e}}-\frac{\chi_{c} i h}{\chi \chi_{e}}$

The capacity of the positive electrode for storing lithium is taken to have a maximum value of $C_{m}$, where this parameter is the maximum number of moles of lithium that can be absorbed per unit volume of the positive electrode. Note that $C_{m}$ is not the maximum capacity of the storage material itself, which can be designated $c_{m}$. If the storage material occupies the volume fraction $f$ of the positive electrode, then $C_{m}=f c_{m}$. The parameter $C_{m}$ is, in principle, measurable. The current concentration of lithium in the positive electrode is designated to be $C$, and a state of charge parameter, $S$, can be introduced such that

$C=S C_{m} \quad 0 \leq S \leq 1$

In the full/empty segment of the positive electrode, $S$ is either zero or 1.

We see that the storage of lithium represents a sink for it in the transport process, and unstorage represents a source, so that, in the active segment of the positive electrode

$\frac{d j}{d x}=-\frac{\partial C}{\partial t}=-C_{m} \frac{\partial S}{\partial t} \quad h+H_{f} \leq x \leq h+H$

The boundary conditions on this equation are Eq. (14) and

$j(h+H, t)=0$

with the latter expressing the fact that lithium ions cannot enter the current collector as it is not an ionic conductor.

For the electrochemical behavior of the positive electrode, we recognize that the flux of lithium into storage particles, measured as moles per unit interface area per unit time, is given by $F(\partial C / \partial t) / a$ where $a$ is the interface area per unit volume of the positive electrode. The interface in question is that between storage material and electrolyte in the composite positive electrode. As a result, the Butler-Volmer equation provides, in the positive electrode, 
$\frac{F}{a} \frac{\partial C}{\partial t}=-i_{o}^{c}\left[\exp \left(\frac{\alpha_{c a} F \eta_{c}}{R T}\right)-\exp \left(-\frac{\alpha_{c c} F \eta_{c}}{R T}\right)\right]$

where $i_{o}^{c}$ is the exchange current density for the interface between storage material and electrolyte in the positive electrode, $\alpha_{c a}$ and $\alpha_{c c}$ are the anodic and cathodic symmetry coefficients for the positive electrode, both positive,

$\eta_{c}=\Phi_{s}(x, t)-\Phi(x, t)-U_{c}$

where

$F U_{c}=\mu^{\text {elyte }}-\mu^{\text {cathode }}$

defines the open circuit potential, $U_{c}$, for the storage material/electrolyte interface in the positive electrode, and $\mu^{\text {cathode }}$ is the chemical potential of lithium stored in the positive electrode.

As noted above, $\mu^{\text {elyte }}$ is a fixed quantity, whereas $\mu^{\text {cathode }}$ will depend on the concentration of lithium in the storage material in the positive electrode, specifically at the surface of the storage particles, and will depend on the stress at that surface as well. However, we have assumed that the concentration of lithium in the storage particles quickly equalizes due to the short diffusion distances involved, and we are neglecting the effect of mechanical stress on the redox reactions and on the transport of lithium. Therefore, in our model, $\mu^{\text {cathode }}$ will depend only on the concentration, $c$, of uniformly distributed lithium within the storage particles. We first assume ideal thermodynamics, which means that $\mu^{\text {cathode }}$ ranges smoothly but exponentially from $-\infty$ to $\infty$ as $c$ rises from 0 to $c_{m}$ [13]. We further simplify the model by taking $\mu^{\text {cathode }}=-\infty$ at $c$ $=0$ and $\mu^{\text {cathode }}=\infty$ at $c=c_{m}$ but having a uniform finite value in between. Thus for any storage particle that is actively storing or unstoring lithium, $U_{c}$ is a finite constant, and thus $U_{c}$ is uniform over active segments of the positive electrode that are engaged in actively storing or unstoring lithium.

The exchange current density, $i_{o}^{c}$, for the positive electrode should also depend on $c$ [16]. However, to simplify the solution we will take $i_{o}^{c}$ to be a constant.

We combine Eq. (18) \& (20) to find the governing equation for the active portion of the positive electrode, namely

$\frac{d j}{d x}=\frac{a i_{o}^{c}}{F}\left[\exp \left(\frac{\alpha_{c a} F \eta_{c}}{R T}\right)-\exp \left(-\frac{\alpha_{c c} F \eta_{c}}{R T}\right)\right] \quad h+H_{f} \leq x \leq h+H$

still subject to the boundary equations (14) \& (19).

Next, we use Eq. (9) \& (23) to write

$\chi_{c} \frac{\partial^{2} \Phi}{\partial x^{2}}+a i_{o}^{c}\left[\exp \left(\frac{\alpha_{c a} F \eta_{c}}{R T}\right)-\exp \left(-\frac{\alpha_{c c} F \eta_{c}}{R T}\right)\right]=0$

subject to the boundary conditions, from Eq. (14) 
$\frac{\partial \Phi\left(h+H_{f}, t\right)}{\partial x}=-\frac{i}{\chi_{c}}$

and from Eq. (19)

$\frac{\partial \Phi(h+H, t)}{\partial x}=0$

We note that Eq. (16) \& (21) enable us to write

$\eta_{c}=-\left(1+\frac{\chi_{c}}{\chi_{e}}\right) \Phi+\Phi_{s}\left(h+H_{f}, t\right)+\frac{\chi_{c}}{\chi_{e}} \Phi_{a}^{e l y t e}-\frac{i(x-h)}{\chi_{e}}-\frac{\chi_{c} i h}{\chi_{e}}-U_{c}$

showing that Eq. (24) is a differential equation in $\Phi$ and $x$ with time implicitly involved due to the presence of $H_{f}$.

Interface between the positive electrode and the current collector As noted above, this interface is impermeable to lithium ions, leading to the condition in Eq. (19). In addition, integration of Eq. (12), subject to boundary condition Eq. (13), a step that has already been partially accomplished, will determine the potential at the current collector. This potential represents the potential difference across the terminals of the battery, as the negative electrode is at zero potential.

\section{Solutions}

Battery in steady state open circuit In this situation the concentration of stored lithium in the positive electrode is uniform and the current density is zero. From Eq. (1) \& (2) we then deduce that

$\Phi_{a}^{\text {elyte }}=-U_{a}$

The equations in the electrolyte, both in the separator and in the positive electrode, to be consistent with zero current density, require that the potential in the electrolyte everywhere is given by Eq. (28), and thus
$\Phi(x, t)=-U_{a}$
$0 \leq x \leq H$

Storage and unstorage of lithium in the positive electrode are in abeyance, and therefore the lefthand side of Eq. (20) is zero, causing Eq. (21), together with Eq. (29), to tell us that

$\Phi_{s}(x, t)=U_{c}-U_{a} \quad h \leq x \leq h+H$

As noted above, this potential is also that of the current collector, and thus is the potential difference across the terminals of the battery.

From Eq. (3) \& (22), we deduce that Eq. (30) leads to 
$\Phi_{S}(x, t)=\frac{1}{F}\left(\mu^{\text {anode }}-\mu^{\text {cathode }}\right) \quad h \leq x \leq h+H$

The chemical potential of lithium in the negative electrode is greater than that in the storage material of the positive electrode [16]. Therefore, $\Phi_{s}(x, t)$ in Eq. (31) is greater than zero. As this is the potential of the current collector, and thus the potential difference between the current collector and the negative electrode, this shows that the current collector is, indeed, at a higher potential than the negative electrode. Hence the terminology of positive electrode and negative electrode that is commonly adopted for the battery, and that we use also.

Note that the potential difference in Eq. (31) is that which would be measured by placing across the battery terminals a calibrated resistor with a very large resistance, and then measuring the current; i.e. by use of a voltmeter.

Solution for a linearized model of the battery The nonlinear nature of the battery behavior makes solution of the system of equations challenging. Therefore, we linearize the Butler-Volmer equations for small surface overpotential magnitudes and proceed to a solution. Bockris et al. [16] comment that the linearized Butler-Volmer result for typical battery systems is accurate, in terms of predicting current density, up to a surface overpotential magnitude of $0.01 \mathrm{~V}$. They also provide a table that shows that the linearized version is even a reasonable approximation, to within about $10 \%$ in terms of current density, up to a surface overpotential magnitude of about $0.05 \mathrm{~V}$.

The linearized Butler-Volmer equation for the interface between the negative electrode and the separator is, from Eq. (1) \& (2),

$i=\frac{i_{o}^{a} \alpha_{a} F \eta_{a}}{R T}=-\frac{i_{o}^{a} \alpha_{a} F\left(\Phi_{a}^{e l y t e}+U_{a}\right)}{R T}$

where

$\alpha_{a}=\alpha_{a a}+\alpha_{a c}$

We rearrange Eq. (32) to obtain

$\Phi_{a}^{\text {elyte }}=-U_{a}-\frac{i R T}{i_{o}^{a} \alpha_{a} F}$

We insert this into Eq. (7) to obtain for the separator

$\Phi(x, t)=-U_{a}-\frac{i R T}{i_{o}^{a} \alpha_{a} F}-\frac{i x}{\chi} \quad 0 \leq x \leq h$

and thus, at the interface between the separator and the positive electrode, from Eq. (8) we deduce that 
$\Phi(h, t)=-U_{a}-\frac{i R T}{i_{o}^{a} \alpha_{a} F}-\frac{i h}{\chi}$

Similarly, in the inactive segment of the positive electrode we deduce from Eq. (10) that

$\Phi(x, t)=-U_{a}-\frac{i R T}{i_{o}^{a} \alpha_{a} F}-\frac{i h}{\chi}-\frac{i(x-h)}{\chi_{c}} \quad h \leq x \leq h+H_{f}$

and from Eq. (11) that

$\Phi\left(h+H_{f}, t\right)=-U_{a}-\frac{i R T}{i_{o}^{a} \alpha_{a} F}-\frac{i h}{\chi}-\frac{i H_{f}}{\chi_{c}}$

We next linearize Eq. (24) to obtain

$\chi_{c} \frac{\partial^{2} \Phi}{\partial x^{2}}+\frac{a i_{o}^{c} \alpha_{c} F \eta_{c}}{R T}=0$

where

$\alpha_{c}=\alpha_{c a}+\alpha_{c c}$

Thereafter we use Eq. (27) \& (34) to convert Eq. (40) to

$\frac{\partial^{2} \Phi}{\partial x^{2}}-\frac{a i_{o}^{c} \alpha_{c} F}{R T}\left(\frac{1}{\chi_{c}}+\frac{1}{\chi_{e}}\right) \Phi=\frac{a i_{o}^{c} \alpha_{c} F}{R T}\left[\frac{i(x-h)}{\chi_{c} \chi_{e}}+\frac{i h}{\chi \chi_{e}}+\frac{i R T}{\chi_{e} i_{o}^{a} \alpha_{a} F}-\frac{1}{\chi_{c}} \Phi_{s}\left(h+H_{f}, t\right)+\frac{U_{a}}{\chi_{e}}+\frac{U_{c}}{\chi_{c}}\right]$

Note that each parameter in the brackets, other than $x$, is a constant, given our assumptions.

The solution to Eq. (42), satisfying all boundary conditions, is

$$
\begin{gathered}
\Phi=\frac{i \lambda \chi_{e}}{\chi_{c}+\chi_{e}}\left[\frac{1}{\chi_{e}}+\frac{1}{\chi_{c}} \cosh \left(\frac{H-H_{f}}{\lambda}\right)\right]\left[\frac{\cosh \left(\frac{x-H_{f}-h}{\lambda}\right)+\frac{\chi_{c}}{\chi_{e}} \cosh \left(\frac{H-H_{f}}{\lambda}\right)}{\sinh \left(\frac{H-H_{f}}{\lambda}\right)}\right] \\
-\frac{i \lambda \chi_{e}}{\chi_{c}+\chi_{e}}\left[\frac{1}{\chi_{c}} \sinh \left(\frac{x-H_{f}-h}{\lambda}\right)+\frac{1}{\chi_{e}} \sinh \left(\frac{H-H_{f}}{\lambda}\right)\right]+\frac{i(h+H-x)}{\chi_{c}+\chi_{e}} \\
+\Phi_{s}(h+H, t)-U_{c}
\end{gathered}
$$

accompanied by 


$$
\begin{gathered}
\Phi_{s}=\frac{i \lambda}{\chi_{c}+\chi_{e}}\left[\frac{\chi_{c}}{\chi_{e}}+\cosh \left(\frac{H-H_{f}}{\lambda}\right)\right]\left[\frac{\cosh \left(\frac{H-H_{f}}{\lambda}\right)-\cosh \left(\frac{x-H_{f}-h}{\lambda}\right)}{\sinh \left(\frac{H-H_{f}}{\lambda}\right)}\right] \\
+\frac{i \lambda}{\chi_{c}+\chi_{e}}\left[\sinh \left(\frac{x-H_{f}-h}{\lambda}\right)-\sinh \left(\frac{H-H_{f}}{\lambda}\right)\right]+\frac{i(h+H-x)}{\chi_{c}+\chi_{e}} \\
+\Phi_{s}(h+H, t)
\end{gathered}
$$

where, in both equations,

$\lambda=\sqrt{\frac{\chi_{c} \chi_{e} R T}{a i_{o}^{c} \alpha_{c} F\left(\chi_{c}+\chi_{e}\right)}}$

and is the characteristic length scale for the solution. These solutions are valid in the active segment of the positive electrode, namely within $h+H_{f} \leq x \leq h+H$. The significance of the term $\Phi_{s}(h+H, t)$ is that it is the potential difference across the terminals of the battery, i.e. the potential of the current collector minus the potential of the negative electrode. It is given by

$$
\begin{aligned}
\Phi_{s}(h+H, t)= & U_{c}-U_{a}-\frac{i h}{\chi}-\frac{i H_{f}}{\chi_{c}}-\frac{i\left(H-H_{f}\right)}{\chi_{c}+\chi_{e}}-\frac{i R T}{i_{o}^{a} \alpha_{a} F}+\frac{i \lambda}{\chi_{c}+\chi_{e}} \sinh \left(\frac{H-H_{f}}{\lambda}\right) \\
& -\frac{i \lambda \chi_{e}\left\{\frac{\chi_{c}}{\chi_{e}}+\left[1+\left(\frac{\chi_{c}}{\chi_{e}}\right)^{2}\right] \cosh \left(\frac{H-H_{f}}{\lambda}\right)+\frac{\chi_{c}}{\chi_{e}} \cosh ^{2}\left(\frac{H-H_{f}}{\lambda}\right)\right\}}{\left(\chi_{c}+\chi_{e}\right) \chi_{c} \sinh \left(\frac{H-H_{f}}{\lambda}\right)}
\end{aligned}
$$

It can be seen that, during discharge, when the current density, $i$, is positive, the potential difference across the battery reduces below the open circuit value, $U_{c}-U_{a}$; i.e. the battery has internal resistance. In Eq. (46) during discharge, the $3^{\text {rd }}$ and $4^{\text {th }}$ terms on the right-hand side are losses in the electrolyte, the $5^{\text {th }}$ term is a coupled loss in the positive electrode due to the electronic conducing material and the electrolyte. The $6^{\text {th }}$ term on the right-hand side is a loss due to the redox reaction at the negative electrode and the last 2 terms are losses from the same source in the positive electrode. During charging, when the current density is negative, the terms just listed play an analogous role generating losses, but now raising the potential difference across the battery above the open circuit value; i.e. due to the losses it takes a higher potential to charge the battery than the theoretical value of $U_{c}-U_{a}$. In both discharge and charging the losses, of course, generate heat.

Solution for a linearized model of the battery with a high internal electron conductivity To help to reduce losses, it is desirable to have a high electron conductivity in the positive electrode, i.e. a high value of $\chi_{e}$. To represent such a situation, we take $\chi_{e}=\infty$. This also simplifies the solution considerably. The potential, $\Phi_{s}$, in the electron conducing material equalizes at the 
potential of the current collector, and the entire solution in $0 \leq x \leq h+H_{f}$ remains unaltered. In the electrolyte in the positive electrode the potential becomes

$$
\Phi=\frac{i \lambda}{\chi_{c}}\left[\operatorname{coth}\left(\frac{H-H_{f}}{\lambda}\right) \cosh \left(\frac{x-H_{f}-h}{\lambda}\right)-\sinh \left(\frac{x-H_{f}-h}{\lambda}\right)\right]+\Phi_{s}(h+H, t)-U_{c}
$$

and the potential difference across the battery is

$\Phi_{s}(h+H, t)=U_{c}-U_{a}-\frac{i h}{\chi}-\frac{i H_{f}}{\chi_{c}}-\frac{i R T}{i_{o}^{a} \alpha_{a} F}-\frac{i \lambda}{\chi_{c}} \operatorname{coth}\left(\frac{H-H_{f}}{\lambda}\right)$

We adopt this special case of the linearized solution henceforth.

Rate of storage and unstorage of lithium in the active region of the positive electrode We note the linearized form of Eq. (20) as

$\frac{\partial C}{\partial t}=-\frac{a i_{o}^{c} \alpha_{c} \eta_{c}}{R T}=-\frac{\chi_{c} \eta_{c}}{\lambda^{2} F}$

The solution then gives us

$\frac{\partial C}{\partial t}=\frac{i}{\lambda F}\left[\operatorname{coth}\left(\frac{H-H_{f}}{\lambda}\right) \cosh \left(\frac{x-H_{f}-h}{\lambda}\right)-\sinh \left(\frac{x-H_{f}-h}{\lambda}\right)\right]$

For $H_{f}=0$, i.e. during the early stages of discharge and charging, Eq. (50) becomes

$\frac{\partial C}{\partial t}=\frac{i}{\lambda F}\left[\operatorname{coth}\left(\frac{H}{\lambda}\right) \cosh \left(\frac{x-h}{\lambda}\right)-\sinh \left(\frac{x-h}{\lambda}\right)\right]$

and consequently

$\frac{\partial C(h, t)}{\partial t}=\frac{i \operatorname{coth}\left(\frac{H}{\lambda}\right)}{\lambda F}$

whereas

$\frac{\partial C(h+H, t)}{\partial t}=\frac{i}{\lambda F \sinh \left(\frac{H}{\lambda}\right)}$

Therefore

$\frac{\partial C(h+H, t) / \partial t}{\partial C(h, t) / \partial t}=\frac{1}{\cosh \left(\frac{H}{\lambda}\right)} \leq 1$

This result shows that positive electrode storage particles near the separator will fill and empty more rapidly than those near the current collector, a commonly known behavior [9-13]. In all 
but positive electrodes that are extremely thin, the difference in rates predicted by Eq. (54) will be considerable, as it is commonly the case that $H \gg \lambda$.

If discharge or charging of the battery commences from, respectively, a uniformly empty positive electrode or a uniformly full one, Eq. (52) can be used to compute the time until storage particles at the separator become, respectively, full or empty. This time is given by

$t_{1}=\frac{C_{m}}{\partial C(h, t) / \partial t}=\frac{\lambda F C_{m}}{i \operatorname{coth}\left(\frac{H}{\lambda}\right)}$

The time taken to completely fill or empty the positive electrode is

$t_{f}=\frac{F C_{m} H}{i}$

Thus,

$\frac{t_{1}}{t_{f}}=\frac{H}{\lambda \operatorname{coth}\left(\frac{H}{\lambda}\right)}$

Given that $H \gg \lambda$, only a small fraction of, e.g. charging time for the battery, will be taken up by filling the positive electrode storage particles near the separator. The balance of the time to charge the battery will be taken up by propagating the inactive segment of the positive electrode through it, i.e. with $H_{f} \neq 0$.

Lithium concentration in the positive electrode Let us consider discharge of the battery commencing at $t=0$ with a positive electrode that is empty of lithium. For the early stage of discharge at constant current density, Eq. (51) leads to

$C(x, t)=\frac{i t}{\lambda F}\left[\operatorname{coth}\left(\frac{H}{\lambda}\right) \cosh \left(\frac{x-h}{\lambda}\right)-\sinh \left(\frac{x-h}{\lambda}\right)\right] \quad 0 \leq t \leq t_{1}$

At time $t=t_{1}$ the inactive segment of the positive electrode appears, in this case saturated with lithium, i.e. henceforth $H_{f} \neq 0$.

Of interest is the rate at which $H_{f}$ propagates. This rate is given by

$\frac{d H_{f}}{d t}=-\frac{\partial C\left(h+H_{f}, t\right) / \partial t}{\partial C\left(h+H_{f}, t\right) / \partial x}$

The numerator for this is given from Eq. (50) by

$\frac{\partial C\left(h+H_{f}, t\right)}{\partial t}=\frac{i \operatorname{coth}\left(\frac{H-H_{f}}{\lambda}\right)}{\lambda F}$

but the denominator can only be obtained by numerical integration. Instead, we consider the situation when $H_{f}=0$, in which case differentiation of Eq. (58) with $t=t_{1}$ provides 
$\frac{\partial C\left(h, t_{1}\right)}{\partial x}=-\frac{C_{m}}{\lambda \operatorname{coth}\left(\frac{H}{\lambda}\right)}$

From Eq. (59)-(61) we then deduce that, at $t=t_{1}$,

$\frac{d H_{f}}{d t}=\frac{i \operatorname{coth}^{2}\left(\frac{H}{\lambda}\right)}{F C_{m}}$

This propagating front will reach the current collector at the time given by Eq. (56) and so, to the neglect of $t_{1}$, which is much smaller, the average velocity of the propagating front is

$\frac{\overline{d H_{f}}}{d t}=\frac{i}{F C_{m}}$

We have previously noted that, typically, $H \gg \lambda$, so that $\operatorname{coth}(H / \lambda) \approx 1$. Thus, the propagating front moves through the positive electrode at a fairly uniform speed. We can expect this to occur during charging as well.

These observations permit us to arrive at an approximate solution for the concentration of lithium in the positive electrode during discharge as a function of position and time. Given what we have ascertained, we can approximate the rate of change of concentration, from Eq. (50), as

$\frac{\partial C}{\partial t}=\frac{i}{\lambda F}\left[\cosh \left(\frac{x-\dot{H}_{f}\left(t-t_{1}\right)-h}{\lambda}\right)-\sinh \left(\frac{x-\dot{H}_{f}\left(t-t_{1}\right)-h}{\lambda}\right)\right]$

where $\dot{H}_{f}$ is a constant rate of propagation of the front, given approximately by Eq. (63). We will give a slightly better approximation below. The approximation in Eq. (64) will be rather good until near the end of discharge, at which stage the hyperbolic cotangent in Eq. (50) will diverge from unity. We integrate Eq. (64) with respect to time and obtain

$C(x, t)=C_{m}\left[\cosh \left(\frac{x-\dot{H}_{f}\left(t-t_{1}\right)-h}{\lambda}\right)-\sinh \left(\frac{x-\dot{H}_{f}\left(t-t_{1}\right)-h}{\lambda}\right)\right]$

with

$\dot{H}_{f}=\frac{i}{F C_{m}\left(1-\frac{\lambda}{H}\right)} \approx \frac{i}{F C_{m}}$

$H_{f}=\dot{H}_{f}\left(t-t_{1}\right)$

This approximate result in Eq. (65) satisfies the initial condition at $t=t_{1}$ given by Eq. (58) and is valid for $t_{1} \leq t \leq t_{f}$ and within $h+H_{f} \leq x \leq h+H$. Though approximate, the result remains consistent until the battery is fully charged.

We note that an equivalent approximation is available for charging the battery, but we have not attempted to derive it. 


\section{Discussion}

A linearized solution for a 1-dimensional solid state lithium-ion battery has been obtained for its charge and discharge. In this discussion section we will address the simplest form of our solution, namely that encompassing Eq. (47) \& (48) for a cell having very good conductivity for electrons in the positive electrode. The length scale, $\lambda$, in that case simplifies to

$\lambda=\sqrt{\frac{\chi_{c} R T}{a i_{o}^{c} F}}$

where we have taken $\alpha_{c}$ to be unity, its theoretical value [16]. The significance of the length $\lambda$ is quite straightforward; it depends on the competition between conductivity of the electrolyte and the rate of the redox reaction that takes lithium into or out of the storage particles. We will describe the situation in discharge, but an equivalent behavior can be identified during charging. If the electrolyte is relatively conductive compared to the rate of the redox reactions, lithium ions are transported relatively rapidly in the electrolyte, so that they move along it in preference to entering the storage particles. As a consequence, a large segment of the electrode is involved in significant rates of storage of lithium into the storage particles, and $\lambda$ is large. In other words, the characteristic features of the solution in the positive electrode are spread out over a long length of it. If the conductivity of the electrolyte is relatively sluggish compared to the rate at which the redox reaction takes place, the lithium ions preferentially enter the storage particles instead of transporting along the electrolyte. Thus, significant storage rates of lithium into the storage particles are confined to a narrow segment of the positive electrode, because almost all lithium that is available for the redox reaction immediately engages in it and little is left to be transported further along the positive electrode. Thus, in this case $\lambda$ is small.

We now consider parametric values of various quantities to determine a typical range for $\lambda$. Ionic conductivity in in electrolytes, $\chi$, can range from $0.1 \mathrm{~S} / \mathrm{m}$ to $0.3 \mathrm{~S} / \mathrm{m}$ (see references in [20]). Since the positive electrode is packed with much storage material, the conductivity of this electrode can be considerably lower than the electrolyte itself. Therefore, we estimate a range for $\chi_{c}$ to be $0.01 \mathrm{~S} / \mathrm{m}$ to $0.3 \mathrm{~S} / \mathrm{m}$. If we consider random close packing of uniform spheres, the volume fraction of storage particles in the positive electrode would be just over $60 \%$, a result that leads to $a=4 / D$ where $D$ is the diameter of the spherical storage particle. Polydispersed particles will have higher volume fraction, while at the same time the presence of electrolyte in the electrode will reduce it. The usually nonspherical shape of particles will increase the area of interface per unit volume. Thus, there is a considerable range for $a$ before even considering the possibilities for an average particle size, which are typically $10 \mu \mathrm{m}$ to $50 \mu \mathrm{m}$. To encompass this situation we will use $a=4 / D$ with $D$ ranging from 10 to $50 \mu \mathrm{m}$.

A known relationship between exchange current density and interface resistance is [21]

$i_{o}=\frac{R T}{F Z}$

where $Z$ is the interface resistance. The length scale further simplifies then to 
$\lambda=\sqrt{\frac{D \chi_{c} Z_{c}}{4}}$

where $Z_{c}$ is the interface resistance for the storage material/electrolyte in the positive electrode.

Values for this resistance, $Z_{c}$, are hard to measure because of the complex shapes of storage particles and their 3-dimensional configuration. Data are available for lithium thiophosphate electrolytes containing NCM-811 storage particles [22], where the resistance of a cell attributable to the electrolyte/storage material interface in the positive electrode is measured to be approximately $300 \Omega$. The cross-sectional area of the battery investigated is approximately $0.8 \mathrm{~cm}^{2}$, the positive electrode is approximately $100 \mu \mathrm{m}$ thick, and its volume fraction of storage material is around $70 \%$. This suggests that there are approximately $15 \times 10^{6}$ storage particles in the positive electrode presenting a total surface area of approximately $50 \mathrm{~cm}^{2}$. If we assume that all particles are spheres and are simultaneously engaged in the redox reaction, the interface resistance $Z_{c}=15 \times 10^{3} \Omega \mathrm{cm}^{2}$. On the other hand, if we assume that only $10^{6}$ particles are ever simultaneously in redox, the interface resistance falls to $10^{3} \Omega \mathrm{cm}^{2}$. Values for resistances between lithium metal electrodes and electrolytes have been found to be as low as $2 \Omega \mathrm{cm}^{2}$ and as high as $50 \Omega \mathrm{cm}^{2}$ with a typical value around $15 \Omega \mathrm{cm}^{2}$ [23-26]. The indium electrode used for the negative position in the cell tested by Koerver et al. [22] has an interface resistance of approximately $30 \Omega \mathrm{cm}^{2}$. Given the low values for negative electrodes, we select values from 1 $\Omega \mathrm{cm}^{2}$ to $10^{3} \Omega \mathrm{cm}^{2}$ for $Z_{c}$.

Given the range of values just quoted, we find that the length scale, $\lambda$, can be as small as $1 \mu \mathrm{m}$ and as high as $500 \mu \mathrm{m}$ or more. Since positive electrodes are typically $100 \mu \mathrm{m}$, we see that the length scale, $\lambda$, may be negligble in comparison; indeed, since storage particles range up to 50 $\mu \mathrm{m}$ and even greater, the length scale may well be small compared to microstructural dimensions. Such small length scales would be associated with positive electrode interfaces with high redox reaction rates. Those interfaces that have more sluggish redox reactions could have a length scale, $\lambda$, that is comparable to or greater than the thickness of the positive electrode.

During discharge of the battery we depict the solution for potential in Figure 2, which is schematic rather than exact. In this case we assume that the length scale, $\lambda$, is comparable to or greater than the thickness of the positive electrode. As noted above, the potential in the negative electrode is set to zero as a datum. As given by Eq. (6) \& (34), at the interface between the electrode and the separator at $x=0$ the potential drops by just over $U_{a}$. We have assumed that the open circuit potential of the negative electrode versus the electrolyte is such that $U_{a}>0$. It is possible for it to be negative, which occurs in cases where the internal chemical potential of lithium ions in metal exceeds its value in the electrolyte. 


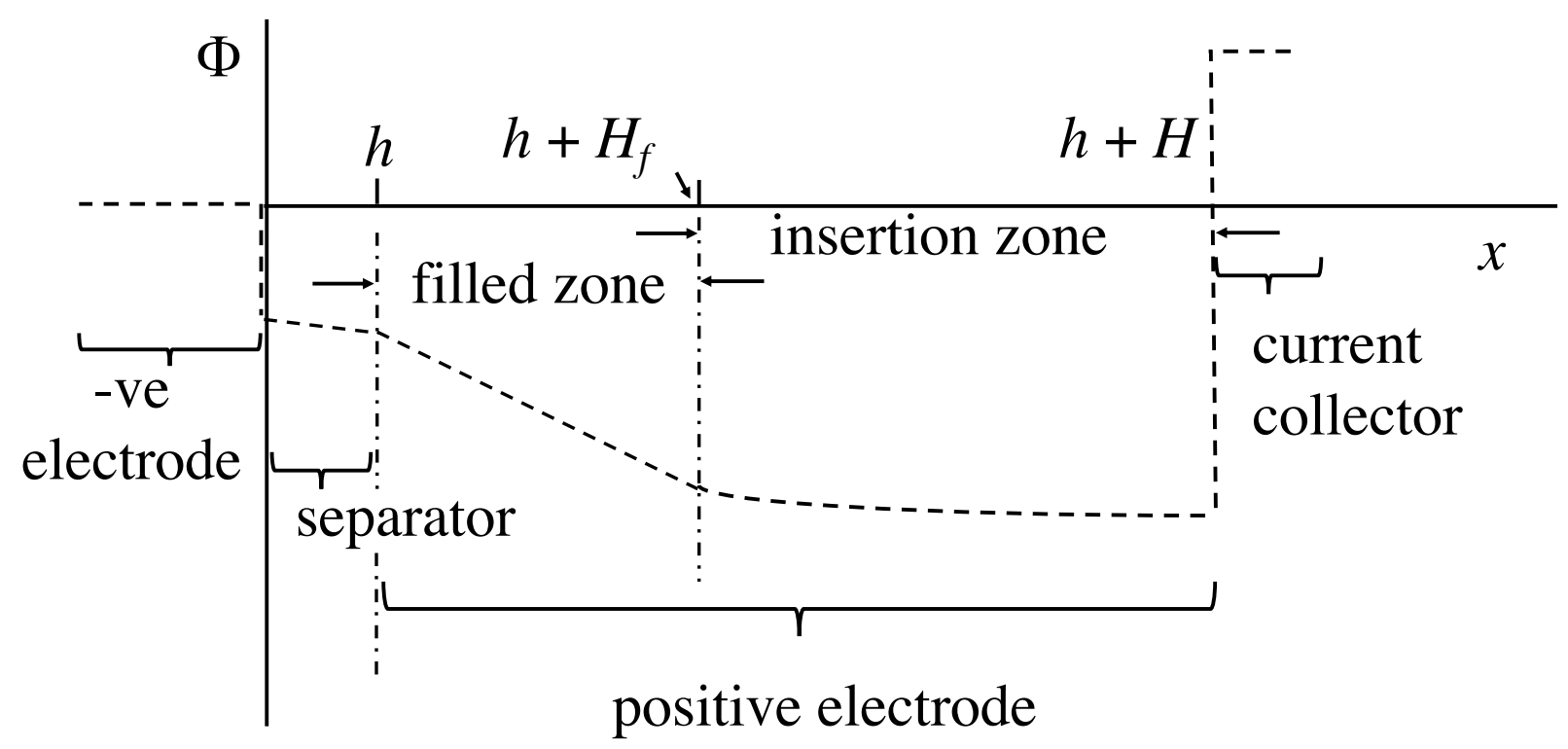

Figure 2: Plot of the potential throughout the battery during discharge, with potential of the negative electrode set to zero as a datum. Losses have been exaggerated for clarity.

The potential falls linearly in the separator in $0 \leq x \leq h$, as given by Eq. (36). The gradient of the potential, i.e. the magnitude of the electric field, is determined by the conductivity of the electrolyte. The behavior in the filled segment of the positive electrode, in $h \leq x \leq H_{f}$ is similar to that in the separator in that the potential falls linearly there as well, as given by Eq. (37). In this segment of the battery the conductivity is generally less than that in the separator, due to the presence of the storage particles. As a consequence, the gradient is steeper, i.e. the magnitude of the electric field is greater.

In the active segment of the positive electrode in $H_{f} \leq x \leq H$, defined as the insertion zone in Figure 2, the solution is spatially nonlinear, as given by Eq. (43) \& (47). This feature is caused by insertion of lithium into the storage particles in this segment of the positive electrode. At the boundary between the filled segment of the electrode and the active segment, at $x=H_{f}$, the gradient of the potential, i.e. the magnitude of the electric field, in the active segment of the positive electrode is equal to that in the filled zone. However, the magnitude of the electric field falls as one moves toward the right in the active segment of the positive electrode, until the electric field is zero at the current collector (at $x=H$ ). The electric field magnitude diminishes in this way because the lithium ion current is decaying, a feature occurs because some of the lithium, at each location, is being inserted into storage particles. The electric field is zero at the current collector because no lithium can enter it, and thus the current composed of lithium ions is zero at this location.

At the current collector (at $x=H$ ) the potential jumps to the value given by Eq. (44) or (48). In the case of a battery with high conductivity for electrons in the positive electrode, i.e. where Eq. (48) prevails, the potential in the current collector gives the potential in storage particles. Therefore, the difference between the potential in the current collector and that in the active segment of the positive electrode in $H_{f} \leq x \leq H$ is somewhat less than $U_{c}$, associated with the 
overpotential at the storage particle surface. Obviously, the potential in the current collector is the potential difference across the terminals of the battery.

As noted previously, the position $x=H_{f}$ sweeps across the positive electrode, i.e. $H_{f}$ is a function of time. Therefore, Figure 2 is a snapshot with discharge of the battery enlarging the filled zone and diminishing the insertion zone.

We now consider the case where the length scale, $\lambda$, is small compared to the thickness of the positive electrode. A consequence of this feature is that the solution in the positive electrode involves significant rates of lithium insertion into and extraction from storage particles in only a thin slice of the electrode. The solution for the potential in the battery in this case is shown in Figure 3, also schematic rather than exact. The features of the solution in this case are largely the same as those shown in Figure 2. The main difference is that the active segment of the positive electrode, designated, as before, as the insertion zone, is dominated by a highly active zone which is relatively narrow. Although some insertion is occurring in the remainder of the positive electrode to the right, the amount of insertion occurring there is small, and so the electric potential is almost uniform in that segment, as shown in Figure 3. Most of the potential drop in the positive electrode takes place in the narrow segment, again as shown in Figure 3, and most of the lithium insertion into storage particles occurs in that narrow segment as well. As a consequence of this feature, the insertion zone, remaining narrow, sweeps across the positive electrode, moving to the right as the battery discharges. The rate at which this occurs, to first order, is constant and given by the rate of discharge of the battery, i.e. at the rate givenby Eq. (63).

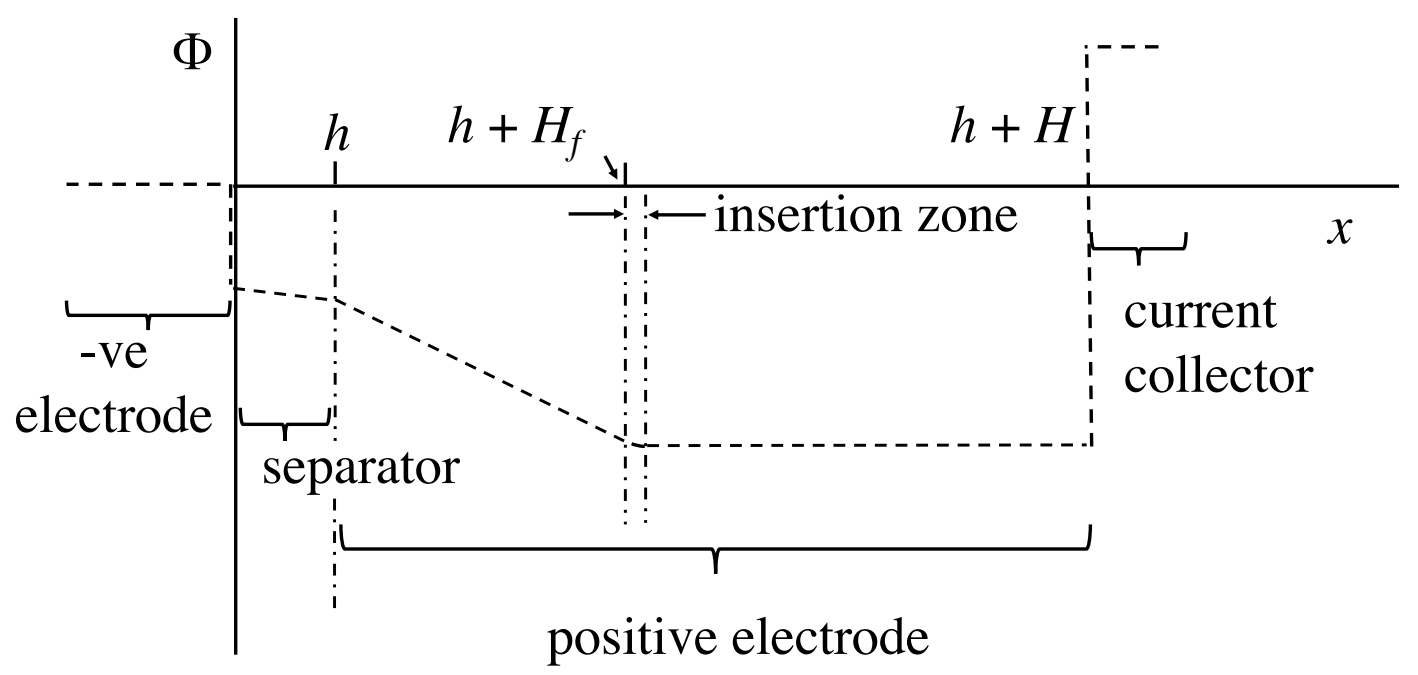

Figure 2: Plot of the potential throughout the battery during discharge. Losses have been exaggerated for clarity. The result shown is for a battery with a very high conductivity for electrons in the positive electrode and a very high rate of lithium insertion into its storage particles. As a result, lithium insertion in the positive electrode occurs only within a very narrow zone. This zone propagates from left to right, effectively as a reaction front. 
Typical values have been given above for the resistances at the interface between the negative electrode and the electrolyte and for the interface between the electrolyte and the storage material. From these data and Eq. (69) the exchange current densities can be computed. For the interface between lithium metal and the electrolyte, where the interface resistance can range from $2 \Omega \mathrm{cm}^{2}$ to $50 \Omega \mathrm{cm}^{2}$, Eq. (69) gives us exchange current densities at $T=300^{\circ} \mathrm{K}$ that range from $0.5 \mathrm{~mA} / \mathrm{cm}^{2}$ to $10 \mathrm{~mA} / \mathrm{cm}^{2}$, given that $R=8.314 \mathrm{~J} / \mathrm{mole}^{\circ} \mathrm{K}$ and $F=96485 \mathrm{C} / \mathrm{mole}$. For the interface between the electrolyte and the storage particles in the positive electrode, we have estimated the interface resistance, in one case, to be possibly anywhere from $10^{3} \Omega \mathrm{cm}^{2}$ to $15 \mathrm{x}$ $10^{3} \Omega \mathrm{cm}^{2}$, and Eq. (69) then gives us exchange current densities at $T=300^{\circ} \mathrm{K}$ that range from $0.002 \mathrm{~mA} / \mathrm{cm}^{2}$ to $0.03 \mathrm{~mA} / \mathrm{cm}^{2}$.

We now note that the status of our linearized solution depends on the relationship between the current densities of operation in the battery and the exchange current densities at the interfaces in it. If the current densities of operation for the battery are similar to, or greatly exceed, the exchange current densities of the interfaces, then our solution is not relevant and the nonlinear version of the Butler-Volmer equation must be used rather than the linearized relationship we have utilized. In contrast, if the current densities of operation are low compared to the exchange current densities at the interfaces, our linearized solution is valid. This situation arises because the redox kinetics occurring at those interfaces will operate at rates that keep them within the linearized regime of the Butler-Volmer equation. This is the case that Bockris et al. [16] describe in regard to linear response of the redox kinetics, but put in context in terms of the magnitudes of the overpotentials that are involved.

Current densities of operation for solid state batteries tend to be in the range $0.1 \mathrm{~mA} / \mathrm{cm}^{2}$ to 5 $\mathrm{mA} / \mathrm{cm}^{2}$ with $2.5 \mathrm{~mA} / \mathrm{cm}^{2}$ being a desirable target. Since exchange current densities between lithium metal electrodes and solid electrolytes, as noted above, range from $0.5 \mathrm{~mA} / \mathrm{cm}^{2}$ to 10 $\mathrm{mA} / \mathrm{cm}^{2}$, it is likely that the battery often will be operating in the linear regime of redox kinetics at this interface. The easiest way to assess whether the linear regime is operative at interfaces in the positive electrode is to use data from the study we utilized to ascertain interface resistance [22]. If we assume that the cell that was tested in that case is operated at a current density of 0.1 $\mathrm{mA} / \mathrm{cm}^{2}$, the total current is $0.08 \mathrm{~mA}$. If such a current simultaneously enters all $15 \mathrm{x} 10^{6}$ storage particles in the positive electrode at the same rate, the current density at the particle surface for $10 \mu \mathrm{m}$ diameters is approximately $0.002 \mathrm{~mA} / \mathrm{cm}^{2}$. Since the exchange current density at this interface is as high as $0.03 \mathrm{~mA} / \mathrm{cm}^{2}$, it seems likely, again, that the positive electrode will be operating in the linear regime of Butler-Volmer kinetics. On the other hand, at the highest current density of operation, $5 \mathrm{~mA} / \mathrm{cm}^{2}$, with only a subset of $10^{6}$ storage particles in the positive electrode receiving the total current, the current density at the surface of the storage particles will be of the order of $0.15 \mathrm{~mA} / \mathrm{cm}^{2}$. Clearly this is beyond the linear regime of redox kinetics for this interface.

Thus, we conclude from the discussion of the linear solution and its status that at the lower end of the desirable range of current densities solid state lithium-ion batteries are likely to operate in the linear regime redox kinetics for both electrodes. At higher rates of operation, the negative electrode is likely to remain in the linear regime of the Butler-Volmer equation, but the positive electrode probably will respond in a nonlinear manner. However, our assessment is based on 
quite limited data for the properties of slid state positive electrodes that are also difficult to interpret. Therefore, there still remains the possibility that exchange current densities for positive electrodes are higher than we have estimated, and that the linear regime of operation has wider validity at higher current densities than we have surmised.

\section{Concluding comment}

An approximate solution is provided for a solid state, 1-dimensional battery with a lithium metal negative electrode and single ion conducting electrolyte. The solution assumes very approximate thermodynamics for the intercalation of lithium in the positive electrode of the battery. We note that the assumption leads to a discrete front that can travel through the battery to divide a completely full or completely empty segment of the positive electrode from a segment that is still actively storing or unstoring lithium. In addition, it seems likely that in some positive electrodes the storage and unstorage of lithium in particles occurs only in a very narrow slice of that electrode and that this slice propagates along the positive electrode as discharge and charging of the battery takes place. This narrow slice of active positive electrode in that case divides it into a segment that will already be full or empty and a segment that has not yet gained or lost any of its lithium. In addition, it is found that at lower current densities of operation in solid state batteries that it will be in the linear regime of and that our solution is then relevant.

\section{Acknowledgement}

As noted above, this contribution is dedicated to Norman Fleck on the occasion of his $60^{\text {th }}$ birthday. I have spent many happy hours in collaboration with Norman, and it has been highly productive, educational, rewarding and enjoyable to do so. I look forward to further interactions with him on the subject of this paper, lithium-ion batteries.

This work was funded by the University of California, Santa Barbara and by the University of Aberdeen.

\section{References}

[1] C. Yang, K. Fu, Y. Zhang, E. Hitz and L. Hu, "Protected lithium-metal anodes in batteries: From liquid to solid," Advanced Materials 291701169 (2017).

[2] G. Bucci, Y.M. Chiang and W.C. Carter, "Formulation of the coupled electrochemical mechanical boundary value problem, with application to transport of multiple charged species," Acta Materialia 104 pp. 33-51 (2016).

[3] Z. Ahmad and V. Viswanathan, "Role of anisotropy in determining stability of electrodeposition at solid-solid interfaces," Physical Review Materials 1055403 (2017).

[4] Z. Ahmad and V. Viswanathan, "Stability of electrodeposition at solid-solid interfaces and implications for metal anodes," Physical Review Letters 119056003 (2017). 
[5] M. Ganser, F. Hildebrand, M. Kamlah and R.M. McMeeking, "Electro-chemo-mechanical transport theory for binary solid electrolytes," submitted Journal of the Mechanics and Physics of Solids (2018).

[6] C. Monroe and J. Newman, "The effect of interfacial deformation on electrodeposition kinetics," Journal of the Electrochemical Society 151 pp. A880-A886 (2004).

[7] C. Monroe and J. Newman, "The impact of elastic deformation on deposition kinetics at lithium/polymer interfaces," Journal of the Electrochemical Society 152 pp. A396-A404 (2005).

[8] P. Goyal and C.W. Monroe, "New foundations for Newman's theory for solid electrolytes: Thermodynamics and transient balances," Journal of the Electrochemical Society 164 pp. E3647-E3660 (2017).

[9] J.S. Newman and K.E. Thomas-Alyea, Electrochemical Systems $3^{\text {rd }}$ Edition, Wiley (2004).

[10] M. Doyle, T.F. Fuller and J. Newman, "Modeling of galvanostatic charge and discharge of the lithium/polymer/insertion cell," Journal of the Electrochemical Society 140 pp. 1526-1533 (1993).

[11] T.F. Fuller, M. Doyle and J. Newman, "Simulation and optimization of the dual lithium ion insertion cell," Journal of the Electrochemical Society 141 pp. 1-10 (1994).

[12] T.F. Fuller, M. Doyle and J. Newman, "Relaxation phenomena in lithium-ion-insertion cells," Journal of the Electrochemical Society 141 pp. 982-990 (1994).

[13] R. Purkayastha and R.M. McMeeking, "A linearized model for lithium ion batteries and maps for their performance and failure," Journal of Applied Mechanics 79 pp. 031021-1 to 031021-16 (2012).

[14] W.H. Woodford, W.C. Carter and Y.-M. Chiang, "Design criteria for electrochemical shock resistant battery electrodes," Energy and Environmental Science 5 pp. 8014-8024 (2012).

[15] L. Porz, T. Swamy, B.W. Sheldon, D. Rettenwander, T. Frömling, H.L. Thaman, S. Berendts, R. Uecker, W.C. Carter and Y.-M. Chiang, "Mechanisms of lithium metal penetration through inorganic solid electrolytes," Advanced Energy Materials 1701003 pp. 1-12 (2017).

[16] J.O’M. Bockris, A.K.N. Reddy and M. Gamboa-Aldeco, Modern Electrochemistry ${ }^{\text {nd }}$. Edition, Vol. 2A Fundamentals of Electrodics Kluwer (2002).

[17] S. Torquato, "Random heterogeneous materials: Microstructure and macroscopic properties," in Interdisciplinary Applied Mathematics, Springer (2002). 
[18] B. Völker and R.M. McMeeking, "Impact of particle size ratio and volume fraction on effective material parameters and performance in solid oxide fuel cell electrodes," Journal of Power Sources 215 pp. 199-215 (2012).

[19] J. Ott, B. Völker, Y. Gan, R.M. McMeeking and M. Kamlah, "A micromechanical model for effective conductivity in granular electrodes," Acta Mechanica Sinica 29 pp. 682-698 (2013).

[20] D. Lin, Y. Liu and Y. Cui, "Reviving the lithium metal anode for high-energy batteries," Nature Nanotechnology 12 pp. 194-206 (2017).

[21] G. Horvai, "Relationship between charge transfer resistance and exchange current density of ion transfer at the interface of two immiscible electrolyte solutions," Electroanalysis 3 pp. 673675 (1991).

[22] R. Koerver, I. Aygün, T. Leichtwei $\beta$, C. Dietrich, W. Zhang, J.O. Binder, P. Hartmann, W.G. Zeier and J. Janek, "Capacity fade in solid-state batteries: Interphase formation and chemomechanical processes in nickel-rich layered oxide cathodes and lithium triphosphate electrolytes," Chemistry of Materials 29 pp. 5574-5582 (2017).

[23] A. Shara, H.M. Meyer, J. Nanda, J. Wolfenstine and J. Sakamoto, "Characterizing the Li 7 La $3 \mathrm{Zr} 2 \mathrm{O} 12$ interface stability and kinetics as a function of temperature and current density," Journal of Power Sources 302 pp. 135-139 (2016).

[24] M. Wang and J. Sakamoto, "Correlating the interface resistance and surface adhesion of the Li metal-solid electrolyte interface," Journal of Power Sources 377 pp. 7-11 (2018).

[25] S. Wenzel, S.J. Sedlmaier, C. Dietrich, W.G. Zeier and J. Janek, "Interfacial reactivity and interphase growth of argyrodite solid electrolytes at lithium metal electrodes," Solid State Ionics 318 pp. 102-112 (2018).

[26] G.B. Appetecchi, S. Scaccia, S. Passerini, "Investigation on the Stability of the LithiumPolymer Electrolyte Interface," Journal of The Electrochemical Society 147 pp. 4448- (2000). 